\title{
Acute piriformis syndrome mimicking cauda equina syndrome: illustrative case
}

\author{
Jan Lodin, MD, ${ }^{1,2}$ Štěpánka Brušáková, MD, ${ }^{3}$ David Kachlík, MD, $\mathrm{PhD},{ }^{4}$ Martin Sameš, MD, $\mathrm{PhD},{ }^{1}$ and Ivan Humhej, MD, $\mathrm{PhD}{ }^{1,4}$ \\ Departments of ${ }^{1}$ Neurosurgery and ${ }^{3}$ Neurology, J.E. Purkyne University, Masaryk Hospital Krajská Zdravotní a.s., Ústí nad Labem, Czech Republic; ${ }^{2}$ Faculty of Medicine in \\ Plzeň, Charles University, Plzeñ, Czech Republic; and ${ }^{4}$ Department of Anatomy, Second Faculty of Medicine in Prague, Charles University, Prague, Czech Republic
}

\begin{abstract}
BACKGROUND This report depicts a rare case of acutely developed urinary retention as well as sensory and motor disturbances caused by formation of a large hematoma within the piriformis muscle, which caused compression of nerves within the suprapiriform and infrapiriform foramina, thus imitating cauda equina syndrome. Although cases of acute lumbosacral plexopathy have been described, this case is the first time both urinary retention and sensory and motor disturbances were present.

OBSERVATIONS The most useful tools for diagnosis of acute piriformis syndrome are detailed patient history, magnetic resonance imaging (MRI) of the pelvic region, and electrophysiological testing performed by an experienced electrophysiologist. As a result of diligent rehabilitation, including physiotherapy and electrostimulation, the patient was able to successfully recover, regardless of acute compression of the sacral plexus that lasted 6 days.

LESSONS Clinicians should actively ask about previous pelvic trauma when taking a patient history in similar cases, especially if the patient is receiving anticoagulation treatment. If MRI of the lumbar spine does not reveal any pathologies, MRI of the pelvic region should be performed. Acute surgical decompression is crucial for preserving neurological function. In similar cases, it is possible to differentiate between spinal cord, cauda equina, and pelvic lesions using electrophysiological studies.
\end{abstract}

https://thejns.org/doi/abs/10.3171/CASE21252

KEYWORDS piriformis syndrome; cauda equina; urinary retention; lumbosacral plexus

This report presents a unique case in which a patient presented with acutely developed urinary retention and sensory and motor disturbances of his lower limbs and genitalia caused by compression of nerves within the infrapiriform and suprapiriform foramina by an intramuscular hematoma within the piriformis muscle. After ruling out cauda equina syndrome (CES), the hematoma was acutely evacuated via a transgluteal approach, resulting in improvement of the patient's neurological function. The main purpose of this report is to inform clinicians of the similarity between acute piriformis syndrome (PS) and CES and act as a diagnostic guide in differentiating between the two clinical entities.

\section{Illustrative Case}

A 55-year-old man with a history of low back pain and ischemic disease of the lower limbs was admitted by his general practitioner with symptoms of left-sided gluteal and inguinal pain. The patient was chronically administered warfarin because he received a femoropopliteal bypass 2 years prior. He had bloody urine and an international normalized ratio of 8.0 ; therefore, warfarin treatment was stopped the previous day. Nonetheless, the general practitioner administered analgesics via an intramuscular injection into the patient's left gluteal region and released the patient. Later that day, the patient was admitted to his regional hospital because of progression of left S1 radicular pain. He received a second intragluteal analgesic injection and was transferred to the department of internal medicine at his local hospital for further care. The next day (hospitalization day 2), the patient developed acral weakness of his left lower limb and difficulty urinating. On hospitalization day 3 , the patient developed urinary retention, erectile dysfunction, saddle hypesthesia, L5 and S1 hypesthesia, and loss of movement from the knee distally. He was transferred back to the regional hospital to rule out CES. Magnetic resonance imaging (MRI) was performed

ABBREVIATIONS CES = cauda equina syndrome; $\mathrm{CMCT}=$ central motor conduction time; $\mathrm{CT}=$ computed tomography; EMG = electromyography; $\mathrm{LSP}=$ lumbosacral plexopathy; $\mathrm{MRI}$ = magnetic resonance imaging; $\mathrm{PS}=$ piriformis syndrome.

INCLUDE WHEN CITING Published October 25, 2021; DOI: 10.3171/CASE21252.

SUBMITTED April 29, 2021. ACCEPTED June 1, 2021.

(c) 2021 The authors, CC BY-NC-ND 4.0 (http://creativecommons.org/licenses/by-nc-nd/4.0/) 

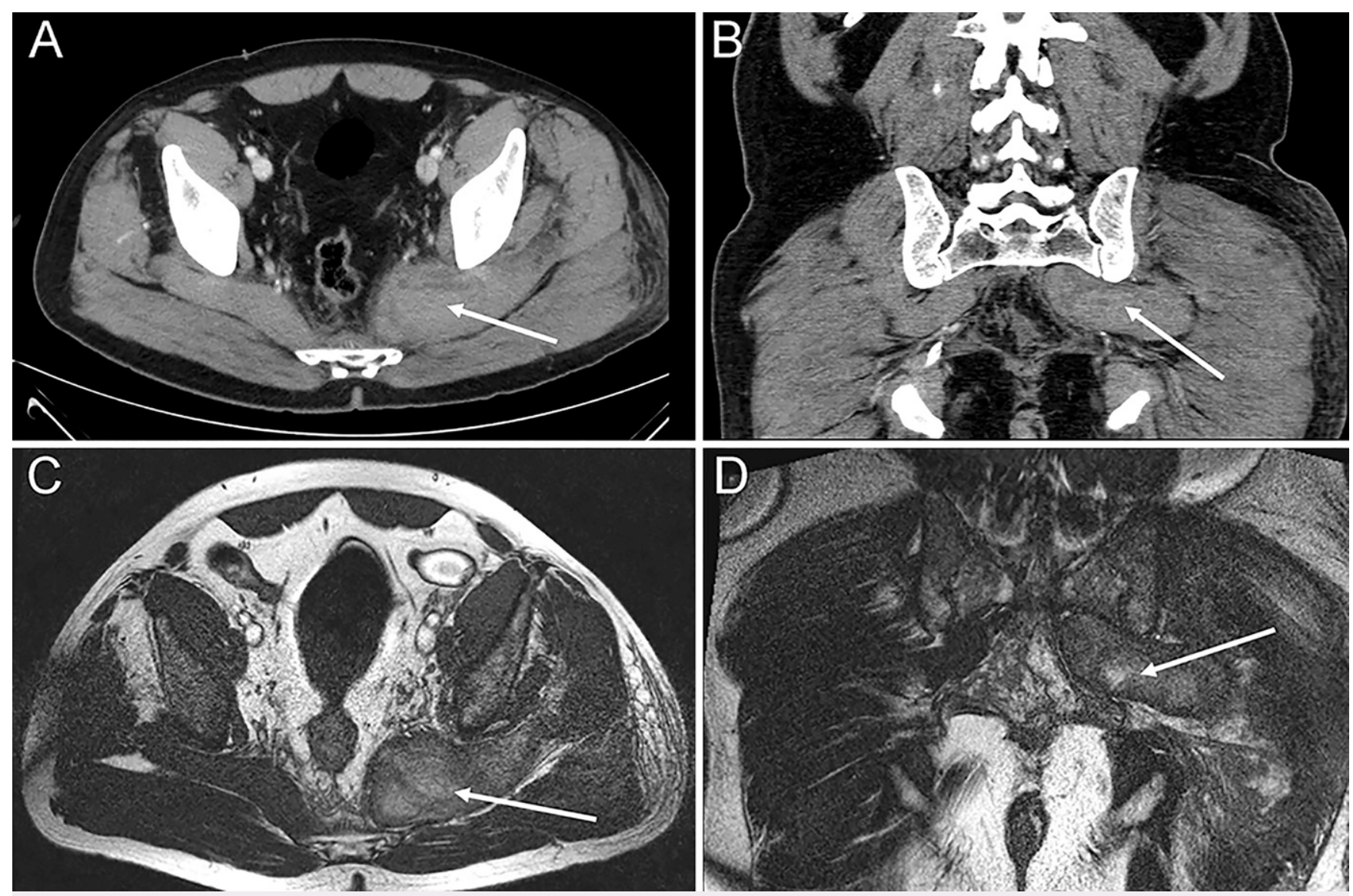

FIG. 1. A: Hematoma within the piriformis muscle on axial preoperative CT (white arrow). B: Hematoma within the piriformis muscle on coronal preoperative CT (white arrow). C: Hematoma within the piriformis muscle on axial preoperative MRI T2 true-FISP sequence (white arrow). D: Hematoma within the piriformis muscle on coronal preoperative MRI true-FISP sequence (white arrow).

on hospitalization day 4 but did not demonstrate compression of neural elements within the vertebral canal. An electrophysiological examination showed signs of sciatic nerve neuropathy in the pelvic region, which led to a follow-up pelvic computed tomography (CT) scan demonstrating enlargement of the piriformis muscle (Fig. $1 \mathrm{~A}$ and B). Our neurosurgical department was consulted on hospitalization day 5; however, because we did not have information on the previously administered gluteal analgesic injections, we suggested performing MRI of the pelvic region to rule out a neoplastic lesion. The MRI result was indicative of a hematoma within the piriformis muscle (Fig. 1C and D). The patient's coagulopathy was corrected, and he was immediately transferred to our department for hematoma evacuation on hospitalization day 6.

Surgery was performed via a transgluteal approach (Fig. 2A), during which the sciatic, inferior gluteal, and posterior femoral cutaneous nerves were identified within the infrapiriform foramen along with a large hematoma within the piriformis muscle (Fig. 2B). The piriformis muscle was incised, the hematoma was evacuated (Fig. $2 \mathrm{C}$ ), and deliberation of the sacral plexus nerves was performed (Fig. 2D). Postoperative MRI of the pelvic region was performed and showed no complications or residual hematoma. During the early postoperative period, the patient's urinary function improved; however, weakness and hypesthesia of the left lower limb remained. He was transferred to his local rehabilitation department to receive intensive rehabilitation, including electrostimulation. Two months later, the patient attended a planned check-up in the outpatient clinic. His urinary and sexual dysfunction had completely resolved and his saddle hypesthesia was improving; however, left-sided L5 and S1 anesthesia remained. His left gluteal muscles were flaccid, without any palpable contractions (Fig. 3A), and he had diffuse weakness of his left leg (hip flexion $4 / 5$, knee extension $4 / 5$, hip extension $0 / 5$, knee flexion $2 / 5$, ankle plantar and dorsal flexion $0 / 5$, ankle eversion and inversion 0/5). Functionally, he was able to walk with the aid of two crutches.

The patient was then regularly followed up for a total of 26 months, during which he regularly attended physiotherapy and electrostimulation. His neurological deficit continued to improve; currently, he is able to walk independently and only uses one crutch for longer distances. He has no urinary or sexual dysfunction, and his sensory deficit has completely resolved. Reinnervation of the gluteal muscles was successful, with active contraction returning within 6 months of the surgery (Fig. 3B). Weakness of his left leg progressively improved (hip flexion and knee extension $5 / 5$, hip extension and knee flexion $4+/ 5$, ankle plantar and dorsal flexion $4 / 5$, ankle eversion and inversion $4 / 5$, toe flexion and extension $3 / 5$ ) (Fig. $3 \mathrm{C}$ and D). The patient is completely ambulatory and able to perform activities of daily living without major limitations. 

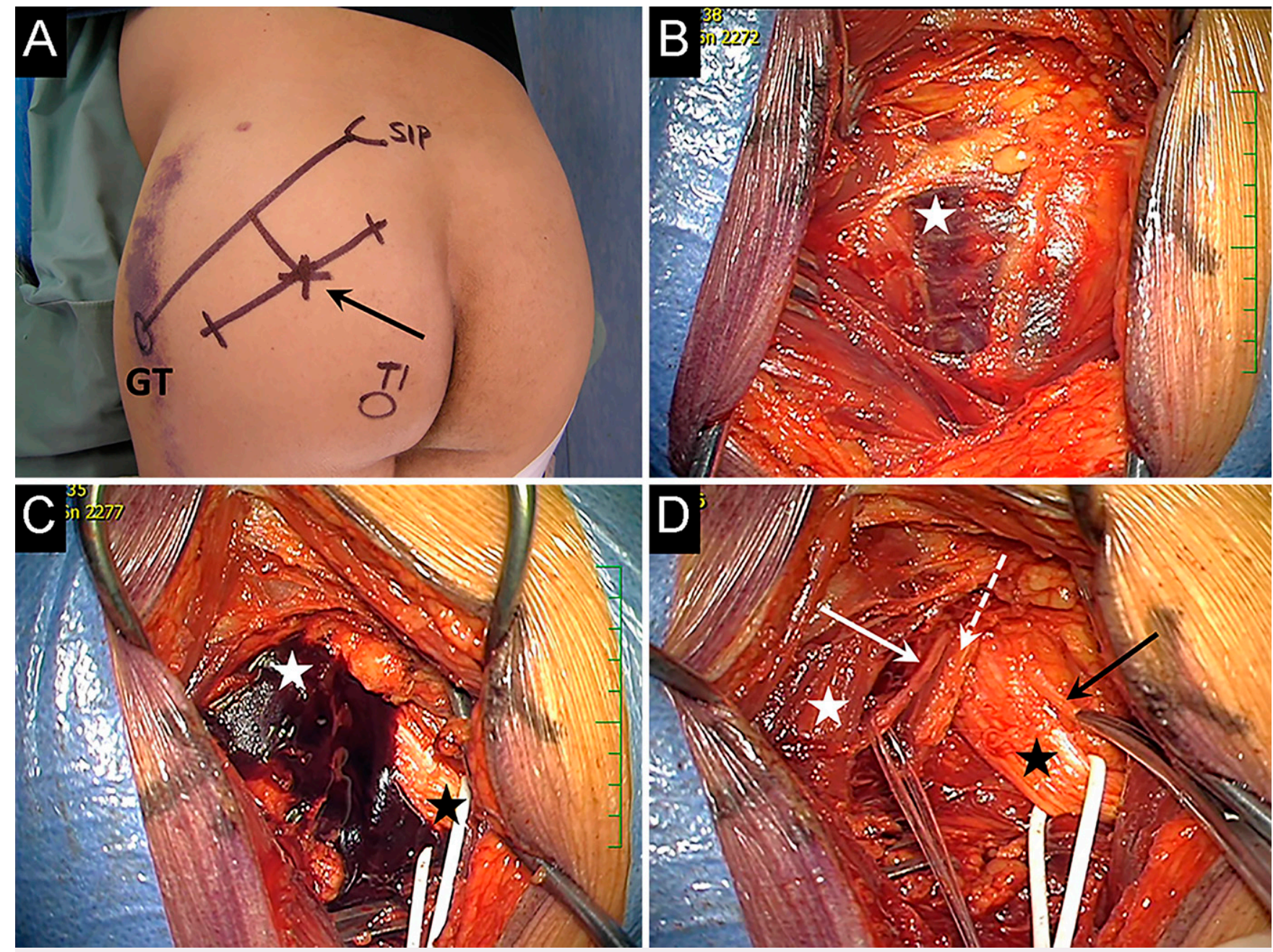

FIG. 2. A: Planning of the transgluteal approach using anatomical landmarks (GT = greater trochanter; SIP = superior posterior iliac spine; $\mathrm{TI}=$ ischial tuberosity) to identify the piriformis muscle and sciatic nerve (black arrow). B: Dark hematoma within the piriformis muscle (white star). C: Evacuation of the hematoma by incising the piriformis muscle (white star), thus decompressing the sciatic nerve (black star). D: Mobilization of the piriformis muscle (white star) and identification of structures within the infrapiriform foramen: sciatic nerve (black star), inferior gluteal nerve (white arrow), posterior femoral cutaneous nerve (black arrow), and inferior gluteal vessels (white dashed arrow).

\section{Discussion}

\section{Observations}

Anatomy and Definition of PS

The piriformis muscle is a pear-shaped muscle that originates from the pelvic surface of the sacral bone and inserts on the greater trochanter of the femur. It passes through the greater sciatic foramen, which it divides into the suprapiriform and infrapiriform foramina. The suprapiriform foramen only contains the superior gluteal nerve and vessels, whereas the infrapiriform foramen contains the inferior gluteal, sciatic, posterior femoral cutaneous, and pudendal nerves as well as the inferior gluteal and internal pudendal vessels. ${ }^{1}$ PS is a term most often used to describe neuropathy of nerves within the infrapiriform foramen caused by piriformis muscle compression. ${ }^{2}$ Most commonly, only the sciatic nerve is affected and the remaining nerves of the surrounding foramina are spared. Rarely, multiple nerves of the lumbar and sacral plexus can be affected; in these cases, we use the clinical term lumbosacral plexopathy (LSP), which is defined as a set of symptoms developing as a result of dysfunction of the anterior rami of the lumbar, sacral, and coccygeal nerves. ${ }^{3}$ Furthermore, at least two different root levels and two different peripheral nerves must be involved on electrophysiological studies. ${ }^{4}$ Clinical symptoms most often include sensory and motor neurological deficits, with urinary and sexual dysfunction in cases in which the lower portion (L4-S4) of the plexus is affected. ${ }^{4}$ Several pathologies can cause lumbosacral neuropathies, including neoplastic lesions, ischemia, internal liac artery aneurysms, trauma, and inflammation. ${ }^{5-8}$ Therefore, the term PS should only be used to describe proximal neuropathies that are directly caused by the piriformis muscle. ${ }^{9}$

PS can be classified based on its etiology as primary or secondary PS. In primary PS, symptoms are caused by anatomical variations of the piriformis muscle, the sciatic nerve, or its trajectory. Secondary PS is caused by the presence of a precipitating factor such as microtrauma, ischemia, neoplasm, hematoma, or infection. ${ }^{10}$ Most cases of PS occur as chronic conditions, which present with sciatica without sensory or motor deficits. ${ }^{11}$ Acute development of PS with progressive 

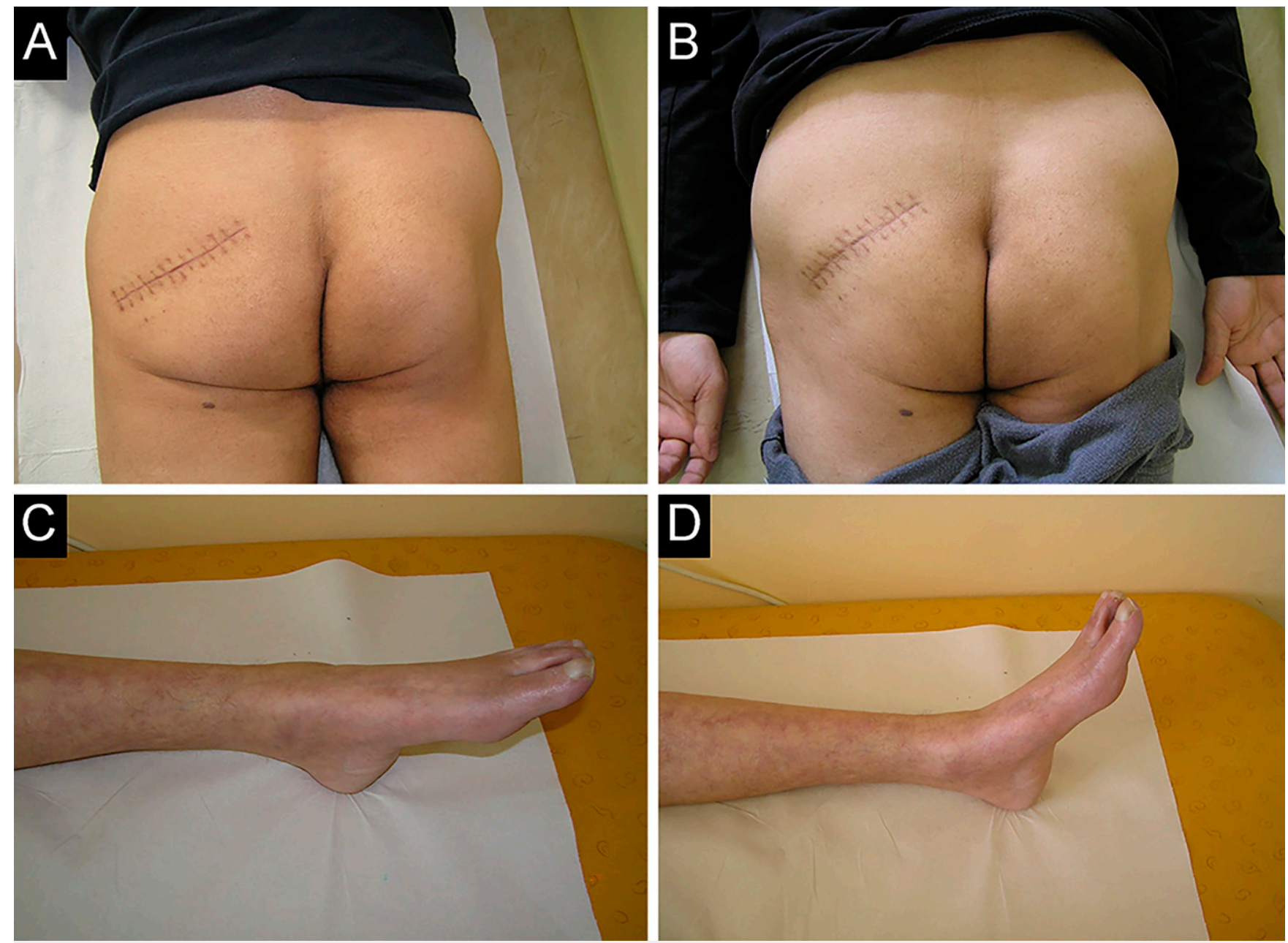

FIG. 3. A: Absent contraction of the left gluteal muscles at the 3-month follow-up. B: Active contraction of the left gluteal muscles at the 6-month followup. C: Maximal plantar flexion at the 26-month follow-up. D: Maximal dorsal flexion and toe extension at the 26-month follow-up.

sensory and motor deficits is rare, with only a handful of cases described in the setting of pyomyositis of the piriformis muscle. ${ }^{12}$ Nonetheless, even among these cases, only $\mathrm{Hu}$ et al. presented a case in which a patient developed urinary retention caused by pyomyositis of the piriformis muscle. ${ }^{13}$ However, their patient did not present with signs of sensory or motor deficits. To the best of our knowledge, no other cases of acute PS resulting in urinary retention have been described.

The case described above presents a rare set of circumstances in which a case of acute secondary PS resulted in LSP due to an aggressively expanding hematoma within the piriformis muscle. The clinical presentation was similar to that of cauda equina compression, for which it was primarily mistaken.

\section{Diagnosis of PS, LSP, and CES}

Diagnosis of PS and LSP, especially in an acute setting, is a difficult feat because their clinical presentations may be similar to the more common CES. As always, the treating clinician should start with an accurate clinical history. Although our patient did have a history of low back pain, the key piece of clinical information was the patient's history of warfarin use combined with multiple intramuscular analgesic injections. Unfortunately, these facts were not initially taken into account at admission, which resulted in a delayed diagnosis of piriformis hematoma while considering CES. Although CES is certainly a more common cause of acute urinary retention with lower limb sensory and motor disturbances than compression of the lumbosacral plexus, a local hematoma should be strongly considered in a patient receiving anticoagulation treatment after local trauma. ${ }^{14}$

The second diagnostic component is clinical examination of the patient. Unfortunately, both CES and LSP have a wide range of clinical symptoms that can present in several combinations, which makes clinical diagnosis of either entity problematic. CES is typically classified as complete or incomplete. Patients with incomplete CES present with various degrees of urinary dysfunction, sciatica, lumbosacral spinal nerve root paresis, and saddle hypesthesia. Conversely, complete CES is characterized by complete urinary retention, absence of bladder control, saddle anesthesia, sciatica, and various degrees of motor weakness of the lower extremities. ${ }^{15}$ The degree of lower limb involvement in both cases is variable and related to the affected motion segment. Low lesions of the lumbar spine (L5-S2) typically involve nerve roots forming the sacral plexus and present with sciatica, hyporeflexia of the L5-S2 segments, 
TABLE 1. Summary of clinical symptoms of the different forms of CES and PS

High Lesion CES (L2-4) Low Lesion CES (L5-S2) Classic PS (sciatic nerve) PS Plus (lumbosacral plexopathy)

Pain

\begin{tabular}{|c|c|c|c|c|}
\hline Leg pain & + & + & + & + \\
\hline Low back pain & + & + & \pm & \pm \\
\hline \multicolumn{5}{|l|}{ Sensory deficit } \\
\hline Saddle anesthesia & + & + & - & \pm \\
\hline Radicular anesthesia & + & + & - & + \\
\hline \multicolumn{5}{|l|}{ Motor deficit } \\
\hline Hip \& thigh muscle groups & + & - & - & - \\
\hline Foot muscle groups & + & + & - & + \\
\hline Bladder dysfunction & + & + & - & + \\
\hline \multicolumn{5}{|l|}{ Hyporeflexia } \\
\hline L2-4 & + & - & - & - \\
\hline L5-S2 & + & + & + & + \\
\hline \multicolumn{5}{|l|}{ Symptom laterality } \\
\hline One-sided & + & + & + & + \\
\hline Bilateral & + & + & - & - \\
\hline
\end{tabular}

$+=$ present; $-=$ absent; $\pm=$ present or absent.

acral lower limb weakness, and hypesthesia. High lesions (L2-4) present with sciatica, hyporeflexia of the L2-S2 segments, global weakness of the lower limb (including hip and thigh muscles), and global lower limb hypesthesia. ${ }^{16}$ CES can present with unilateral or bilateral lower limb symptoms based on anatomy of the lesion, with unilateral symptoms associated with improved prognosis. ${ }^{17}$

Patients with PS classically present with symptoms of sciatica because the sciatic nerve is in close relation to the piriformis muscle within the infrapiriform foramen. Motor or sensory disturbances are rare, and symptoms are thought to be caused by local swelling or inflammation of the piriformis muscle, which then irritates the sciatic nerve. ${ }^{18}$ Rarely, in cases of severe edema and mass effect of the piriformis muscle, multiple nerves of the surrounding foramina can be affected. Clinical sequelae of this compression are neuropathies of the sacral plexus nerves, which manifest as gluteal muscle weakness (superior and inferior gluteal nerves); posterior thigh, calf, and foot muscle weakness (sciatic nerve and its branches); urinary and sexual dysfunction (pudendal nerve); and sensory changes of the posterior thigh (posterior femoral cutaneous nerve), calf, and foot (sciatic nerve branches). Nerves derived from the upper lumbar plexus (T12-L4) are typically spared, and as such, the hip flexor, adductor, and knee extensor muscles are usually fully functional (with the exception of the tensor fasciae latae muscle innervated by the superior gluteal nerve). These sequelae can help differentiate PS from CES caused by a high lesion.

PS also typically affects one side; therefore, the presence of bilateral symptoms suggests CES. Table 1 presents a summary of clinical symptoms of CES and PS and their forms. In the case above, the patient's symptoms were the result of compression of all nerves within the infrapiriform and suprapiriform foramina, which made the diagnosis of PS clinically indistinguishable from CES caused by a low lesion.

The third diagnostic component involves imaging studies. MRI of the lumbar spine should be performed in all patients with newly diagnosed sphincter or lower limb dysfunction to rule out a lesion of the lumbar spine. Unfortunately, in our case, lumbar MRI was performed 2 days after the patient developed muscle weakness and 1 day after developing urinary retention. Furthermore, if lumbar MRI does not demonstrate a pathology explaining the patient's symptoms, MRI of the pelvic region should be promptly performed to rule out pathologies of the lumbosacral plexus. Again, in our case, the diagnostic process was not optimal and the patient received a pelvic CT scan before MRI, which prolonged the diagnostic phase by a further 24 hours. An alternative radiological examination is soft-tissue ultrasound of the gluteal region; however, this is a targeted examination that must be performed to specifically examine the piriformis muscle.

The final diagnostic component is electrophysiological examination of the patient. Although not regularly used in an acute setting, electrophysiological studies can provide diagnostic clues as to the level of neurological injury, if performed correctly. Intramuscular electromyography (EMG) studies are perhaps least useful because signs of denervation, such as fibrillation potentials, positive sharp waves, or complex repetitive charges, are not present in the acute phase. Furthermore, anticoagulant therapy is a relative contraindication to intramuscular studies. Surface EMG is somewhat more useful. PS is classically diagnosed by detecting changes in amplitude and latency of the H-reflex, which are accentuated if compared in a neutral and stressful (flexion, adduction, and internal rotation) position of the hip. ${ }^{19}$ Unfortunately, these changes are also present in CES and therefore cannot be used to differentiate these two pathologies. The optimal method of differentiating PS from CES was suggested by Di Lazzaro et al., who performed double determination of the central motor conduction time (CMCT), first as the difference between response latencies of cortical and paravertebral magnetic stimulation $[\mathrm{CMCT}(\mathrm{m})]$ and second as the difference between the latency of cortical motor evoked potential and the total peripheral conduction time calculated via F-wave latency $[\mathrm{CMCT}(\mathrm{f})] .{ }^{20}$ Patients 
TABLE 2. Electrophysiological changes in CES, PS, and spinal cord lesions

\begin{tabular}{lccc}
\hline & CES & PS & Spinal Cord Lesions \\
\hline Cortical stimulation latency & A & A & A \\
\hline Peripheral conduction time & N & A & N \\
\hline CMCT $(m)^{*}$ & A & N & A \\
\hline CMCT $(\mathrm{f})^{\dagger}$ & N & N & A \\
\hline F-wave latency & A & A & N \\
\hline
\end{tabular}

$\mathrm{A}=$ abnormal; $\mathrm{CT}=$ central time; $\mathrm{DML}=$ distal motor latency; $\mathrm{N}=$ normal; $\mathrm{PT}=$ peripheral time; TPT $=$ total peripheral conduction time.

${ }^{*} \mathrm{CMCT}(\mathrm{m})=\mathrm{CT}-\mathrm{PT}$.

${ }^{\dagger} \mathrm{CMCT}(\mathrm{f})=\mathrm{CT}-\mathrm{TPT}(\mathrm{F}$ wave latency $-1+\mathrm{DML}) / 2$.

with CES typically have abnormal tibial nerve F-wave latencies and $\mathrm{CMCT}(\mathrm{m})$ along with normal $\mathrm{CMCT}(\mathrm{f})$, whereas patients with PS present with abnormal tibial F-wave latencies with $\mathrm{CMCT}(\mathrm{m})$ and CMCT(f) within normal ranges (Table 2). The ability of electrophysiological studies to differentiate between PS and CES makes them crucial diagnostic methods. Our case supports this statement because electrophysiological examination of the patient first suggested a pelvic lesion, which then resulted in graphic diagnosis of the hematoma.

\section{Patient Outcome}

In our case, compression of nerves within the suprapiriform and infrapiriform foramina occurred for a total of 6 days before surgical decompression. Nevertheless, the patient's urinary function improved immediately after surgery, and his motor and sensory function progressively improved for a period of 2 years as a result of regular physiotherapy and electrostimulation. Currently, the patient is fully ambulatory, is able to walk independently, and only has a minor residual motor and sensory deficit. The degree of recovery suggests that acute lesions of the lumbosacral plexus may have better prognosis than acute CES. In a recent review, Kapetanakis et al. stated that if operated within 48 hours of development, urinary function improves in $70 \%$, motor function in $75 \%$, and sensory deficits in $56 \%$ of CES cases. ${ }^{15}$ However, the specific degree of improvement is not mentioned. Unfortunately, a comparison in recovery between patients with CES and PS cannot be performed because a cohort of patients with acutely developed PS with sensory and motor deficits does not exist.

Our theory explaining the high degree of improvement of our patient is that the sciatic nerve may be more resistant to injury compared to nerve roots of the cauda equina. The reason for this may be its large diameter at the level of the infrapiriform foramen $(15.55 \mathrm{~mm}$ on average), based on a metanalysis by Tomaszewski et al., compared to the average diameter of intradural L1-5 nerve roots, which ranges from 3.46 to $4.20 \mathrm{~mm}$ based on a study by Leng et al. ${ }^{21,22}$ The larger diameter of the sciatic nerve means that it also contains a greater proportion of internal epineural fibrous tissue as well as large amounts of collagen I contained within the epineurium. These histological factors have been suggested as reasons for decreased durability of the common fibular nerve compared to the tibial nerve by Schraut et al. ${ }^{23}$ Another crucial factor likely responsible for the patient's recovery is diligent physiotherapy and electrostimulation, which were carried out for a total of 26 months. Because the sciatic nerve lesion was located in the gluteal region, its subsequent regeneration to the most acral leg muscles would be expected to take approximately 26 months (with an approximate length of regeneration $800 \mathrm{~mm}$ at a of pace 1-3 $\mathrm{mm} /$ day). We believe that continuous rehabilitation throughout the regeneration period is crucial in achieving a nerve's maximal regenerative potential.

\section{Lessons}

In conclusion, although CES is the most common cause of urinary dysfunction in conjunction with radicular pain and motor and sensory neuropathy, acute PS can present with a similar set of symptoms. Therefore, a detailed patient history is crucial in identifying risk factors potentially leading to PS. Furthermore, if patients have negative results on lumbar MRI, MRI of the pelvic region should be promptly performed to rule out a lesion of the lumbosacral plexus. Electrophysiological testing can serve as a supportive tool if performed by an experienced neurologist. In cases in which surgical decompression of the lumbosacral plexus is performed, a lengthy time can be expected to pass before complete regeneration has occurred. During this time, we believe that diligent physiotherapy, including electrostimulation, is crucial in achieving an optimal result.

\section{References}

1. Stranding S. Gray's Anatomy: The Anatomical Basis of Clinical Practice. 42nd ed. Elsevier; 2020.

2. Parlak A, Aytekin A, Develi S, Ekinci S. Piriformis syndrome: a case with non-discogenic sciatalgia. Turk Neurosurg. 2014;24(1):117-119.

3. Dydyk AM, Hameed S. Lumbosacral Plexopathy. StatPearls; 2021.

4. Dyck PJ, Thaisetthawatkul P. Lumbosacral plexopathy. Continuum (Minneap Minn). 2014;20(5 Peripheral Nervous System Disorders):1343-1358.

5. Planner AC, Donaghy M, Moore NR. Causes of lumbosacral plexopathy. Clin Radiol. 2006;61(12):987-995.

6. Abdelhamid MF, Sandler B, Awad RW. Ischaemic lumbosacral plexopathy following aorto-iliac bypass graft: case report and review of literature. Ann R Coll Surg Engl. 2007;89(5):W12-3.

7. Ozkavukcu E, Cayli E, Yağci C, Erden I. Ruptured iliac aneurysm presenting as lumbosacral plexopathy. Diagn Interv Radiol. 2008;14(1):26-28.

8. Tung TH, Martin DZ, Novak CB, Lauryssen C, Mackinnon SE. Nerve reconstruction in lumbosacral plexopathy. Case report and review of the literature. J Neurosurg. 2005;102(1 suppl):86-91.

9. Campbell WW, Landau ME. Controversial entrapment neuropathies. Neurosurg Clin N Am. 2008;19(4):597-608, vi-vii.

10. Boyajian-O'Neill LA, McClain RL, Coleman MK, Thomas PP. Diagnosis and management of piriformis syndrome: an osteopathic approach. J Am Osteopath Assoc. 2008;108(11):657-664.

11. Hopayian K, Song F, Riera R, Sambandan S. The clinical features of the piriformis syndrome: a systematic review. Eur Spine J. 2010;19(12):2095-2109.

12. Siddiq MAB, Rasker JJ. Piriformis pyomyositis, a cause of piriformis syndrome. A systematic search and review. Clin Rheumatol. 2019;38(7):1811-1821.

13. Hu MT, Shaw CE, Evans S, Britton TC. Acute sciatica with an infective cause. J R Soc Med. 1998;91(2):87-88.

14. Billet M, Windsor TA. Urinary retention. Emerg Med Clin North Am. 2019;37(4):649-660.

15. Kapetanakis S, Chaniotakis C, Kazakos C, Papathanasiou JV. Cauda equina syndrome due to lumbar disc herniation: a review of literature. Folia Med (Plovdiv). 2017;59(4):377-386.

16. Long B, Koyfman A, Gottlieb M. Evaluation and management of cauda equina syndrome in the emergency department. Am J Emerg Med. 2020;38(1):143-148. 
17. Fraser S, Roberts L, Murphy E. Cauda equina syndrome: a literature review of its definition and clinical presentation. Arch Phys Med Rehabil. 2009;90(11):1964-1968.

18. Niu CC, Lai PL, Fu TS, Chen LH, Chen WJ. Ruling out piriformis syndrome before diagnosing lumbar radiculopathy. Chang Gung Med J. 2009;32(2):182-187.

19. Najdi H, Mouarbes D, Abi-Akl J, Karnib S, Chamsedine AH, Jawish R. EMG in piriformis syndrome diagnosis: reliability of peroneal $\mathrm{H}-$ reflex according to results obtained after surgery, Botox injection and medical treatment. J Clin Neurosci. 2019;59:55-61.

20. Di Lazzaro V, Pilato F, Oliviero A, Saturno E, Dileone M, Tonali PA. Role of motor evoked potentials in diagnosis of cauda equina and lumbosacral cord lesions. Neurology. 2004;63(12):2266-2271.

21. Leng L, Liu L, Si D. Morphological anatomy of thoracolumbar nerve roots and dorsal root ganglia. Eur J Orthop Surg Traumatol. 2018;28(2):171-176.

22. Tomaszewski KA, Graves MJ, Henry BM, et al. Surgical anatomy of the sciatic nerve: a meta-analysis. J Orthop Res. 2016;34(10): 1820-1827.

23. Schraut NB, Walton S, Bou Monsef J, et al. What protects certain nerves from stretch injury? Anat Rec (Hoboken). 2016; 299(1):111-117.

\section{Disclosures}

The authors report no conflict of interest concerning the materials or methods used in this study or the findings specified in this paper.

\section{Author Contributions}

Conception and design: Humhej, Lodin, Sameš. Acquisition of data: Humhej, Lodin. Analysis and interpretation of data: Humhej, Brušáková, Kachlík. Drafting the article: Lodin, Brušáková, Kachlík. Critically revising the article: Humhej, Brušáková, Kachlík, Sameš. Reviewed submitted version of manuscript: Humhej, Brušáková, Kachlík. Approved the final version of the manuscript on behalf of all authors: Humhej. Administrative/technical/material support: Sameš. Study supervision: Sameš.

\section{Correspondence}

Ivan Humhej: J.E. Purkyne University, Masaryk Hospital, Ústí nad Labem, Czech Republic. ivan.humhej@kzcr.eu. 